\title{
MONTE CARLO INVESTIGATION OF DILUTED ANTIFERROMAGNETS IN HIGH MAGNETIC FIELDS
}

\author{
C. Dekker, B.J. Dikken and A.F.M. Arts
}

\author{
Fysisch Laboratorium, Rijksuniversiteit P.O. Box 80.000, 3508 TA Utrecht, The Netherlands
}

(Received 17 December 1984 by R.A. Cowley)

\begin{abstract}
Diluted two- and three-dimensional Ising antiferromagnets, cooled in high external magnetic fields are examined with a Monte Carlo method. In both dimensions stable domain states are observed, which are caused by energy gain of the domain walls at high fields rather than by generated random fields.
\end{abstract}

DILUTED antiferromagnets in an external field have attracted much interest in recent years as they provide an experimental realization of the random-field Ising model (RFIM), i.e. the Ising ferromagnet with quenched site-random fields. The RFIM is the subject of most theoretical work [1], including various Monte Carlo (MC) studies [2-4], whereas field-cooled diluted antiferromagnets allow experimental study of random fields as both systems are believed to be equivalent [5-7]. However, this equivalence is complicated by additional random-exchange effects and moreover by the presence of non-magnetic ions which may provide soft paths along which the domain walls are positioned preferrably. As an extensive theoretical description of the fieldcooled diluted antiferromagnet is lacking, it seems attractive to study this system with a MC method. In this Communication we present a MC investigation of diluted antiferromagnets cooled in high external magnetic fields.

Our results were obtained with a conventional Metropolis MC method on typically $40 \times 40$ square and $14 \times 14 \times 14$ simple-cubic lattices. Periodic boundary conditions were applied. The concentration of spins $\left(S^{z}= \pm 1\right)$ in the $D$-dimensional lattice was chosen midway the percolation threshold and $x=1$, i.e. $x_{2 D}=0.80$ and $x_{3 D}=0.65$. The Hamiltonian for a diluted Ising antiferromagnet in a magnetic field $H_{0}$ is

$$
\begin{aligned}
\mathscr{H} & =-J \sum_{\langle i, j\rangle} \xi_{i} \xi_{j} S_{i}^{z} S_{j}^{z}-H_{0} \sum_{i} \xi_{i} S_{i}^{z} \\
& -H_{a} \sum_{i} \xi_{i} \sigma_{i} S_{i}^{z},
\end{aligned}
$$

where the summation $\langle i, j\rangle$ runs over nearest neighbours, $\xi_{i}=1$ if a site is occupied and $\xi_{i}=0$ if not, and $J$ is the exchange constant. The small symmetry-breaking staggered anisotropy field $H_{a} \sigma_{i}\left(\sigma_{i}= \pm 1\right)$ was added in order to remove the degeneracy of the ground state of the zero-field cooled system. Above the Néel temperature $T_{N}$ this field was set at $H_{a}=5 \times 10^{-3}|J|$, while it vanished below $T_{N}$ according to $H_{a}(T)=H_{a}$ $\exp \left[-10\left(1-T / T_{N}\right)\right]$. In all cases $H_{a}$ was much weaker than the external fields used. This choice of $H_{a}$ ensured that by cooling in zero field the spins were always aligned along the direction of $H_{a}$. On the other hand $H_{a}$ did not affect the transition temperatures which are $T_{N, 2 D}=(1.65 \pm 0.05)|\mathrm{J}|$ and $T_{N, 3 D}=(2.57 \pm 0.06) \mid \mathrm{J}$. This procedure is analoguous to the one employed for the RFIM by Andelman et al. [4]. In all MC runs the system was cooled slowly from the paramagnetic phase with temperature steps of typically $0.05|J|$. At each temperature the new MC equilibrium was built with a time $t_{\text {eq }}$ of about $1400 \mathrm{MC}$ steps. This $t_{\text {eq }}$ was tested to be sufficient except within a small region near $T_{N}$. Longer $t_{\text {eq }}$ (up to $10,000 \mathrm{MC}$ steps) yielded identical results. At each temperature we calculated the sublattice magnetization, the staggered susceptibility, the total energy, and the magnetic specific heat. All results were confirmed by runs on larger lattices (up to $70 \times 70$ and $20 \times 20 \times 20$ ). In order to assure that the findings were not induced by an anomalous lattice configuration, the cooling process was carried out on typically four different configurations.

High fields are needed to obtain sizeable domains within our finite samples. After cooling the sample in a high field $\left(H_{0}>|J|\right.$ in $2 D$ and $H_{0}>2|J|$ in $\left.3 D\right)$ the low-temperature state appears to be disordered showing domains separated by domain walls. A typical example of a $2 D$ domain structure is shown in Fig. 1. These domain states are stable at low temperatures and are always energetically favourable as compared to the single-domain states by typically a few percent. Different runs on the same samples result in the same domain structures at low temperatures with only minor changes in the wall positions. Inspection of the domain walls reveals that these meander mainly over the broken bonds. Furthermore, when no broken bonds are available, neighbouring spins on both sides of the wall are directed along the applied field, i.e. the walls are primarily along the diagonal directions of the lattice. 


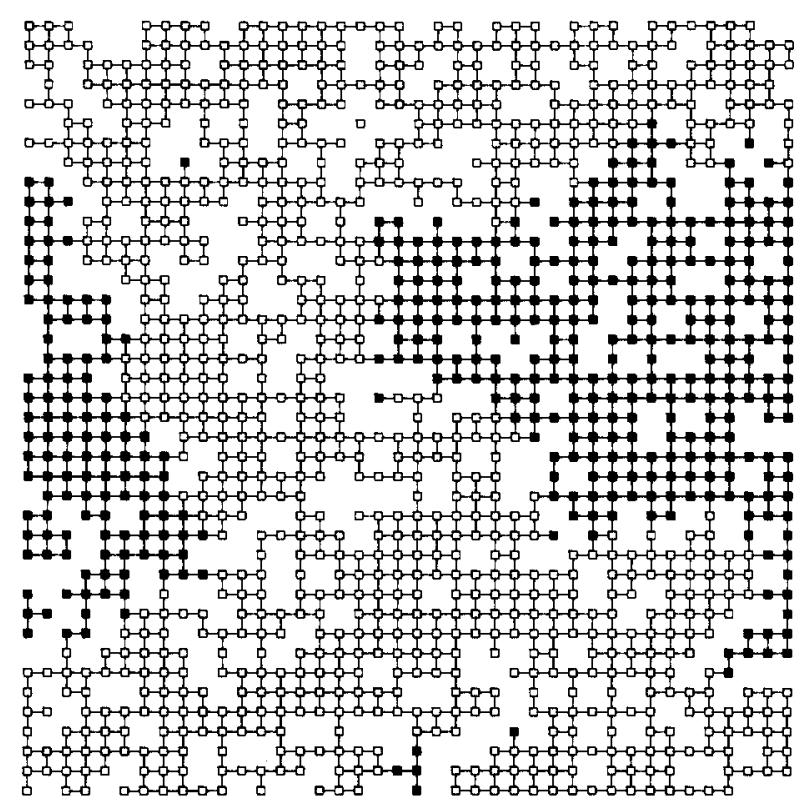

Fig. 1. Example of a domain state obtained by cooling in a field $H_{0}=1.2|J|$ from the paramagnetic phase to $T=0.05|J|$. Full and open squares denote spins in different domains. Solid lines represent nearest-neighbour exchange bonds.

When lower external fields are applied, mostly single-domain states are observed; however the register of the spins is such that they are pointed equally often parallel as antiparallel to the anisotropy field. In some runs energetically unfavourable domain states do appear which freeze in at low temperatures. These states are not reproduced by repeating the cooling process using other random numbers on the same sample. Again no significant differences are observed between the results in $2 D$ and $3 D$.

The temperature dependence of the specific heat is presented in Fig. 2 for the pure and the diluted $3 D$ systems at various fields. In the pure system a sharp asymmetric peak, characteristic for the $3 D$ Ising transition [8], is observed, which shifts towards lower temperatures with increasing fields. The Néel temperature $T_{N}\left(H_{0}\right)$, inferred from the maximum in the specific heat, varies like $T_{N}\left(H_{0}\right)=T_{N}(0)-b H_{0}^{2}$ with $b=$ $(0.054 \pm 0.003) /|J|$. This value is remarkably close to the mean-field result $b=\left[4 T_{N}(0)\right]^{-1}=(0.0559 \pm 0.0005) / V J$ obtained for small $H_{0}$, when for $T_{N}(0)$ the experimental transition temperature is taken. Quite different behaviour is found in the diluted samples. Appreciable broadening of the shape of the specific heat is observed for fields larger than $1.5|J|$. The position of the maximum is gradually lowered, but not to the same extent as in the pure system. The same behaviour is observed for the staggered susceptibility. Similar findings are obtained in the $2 D$ systems. For the undiluted $2 D$ samples we find $b=(0.11 \pm 0.02) /|J|$, which again agrees with the mean-field result $b=(0.109 \pm 0.003) /|J|$.

Combining the findings for the thermodynamic properties and the low-temperature states it appears that high magnetic fields break up the long-range order in diluted antiferromagnets. However, one must be careful in interpreting this in terms of random-field effects since the break-up has only been observed for fields at which Zeeman and exchange energies are comparable in magnitude. For instance, at a field $\left.H_{0}\right\rangle|J|$ spins connected to the infinite cluster by only one exchange bond will be directed along the field. Similarily, at these fields it becomes favourable to pin a domain wall to a spin with two magnetic nearest neighbours. For fields $H_{0}>2|J|$, also spins with three magnetic neighbours may act as pinning centra for the walls. Inspection of the topology of the diluted magnetic lattice shows that even at a concentration far from the percolation threshold, pathes can be constructed which entail a large number of these pinning sites. For example in Fig. 1, which is obtained for $H_{0}=1.2|J|$, the number of pinning sites (with two bonds) in the domain wall is about six times the number expected for a randomly situated wall. Thus a domain wall will lower its energy by meandering over these sites and the vacancies. At sufficiently high fields it even becomes energetically advantageous to create walls and the system will enter a multi-domain state. Essentially these effects have been

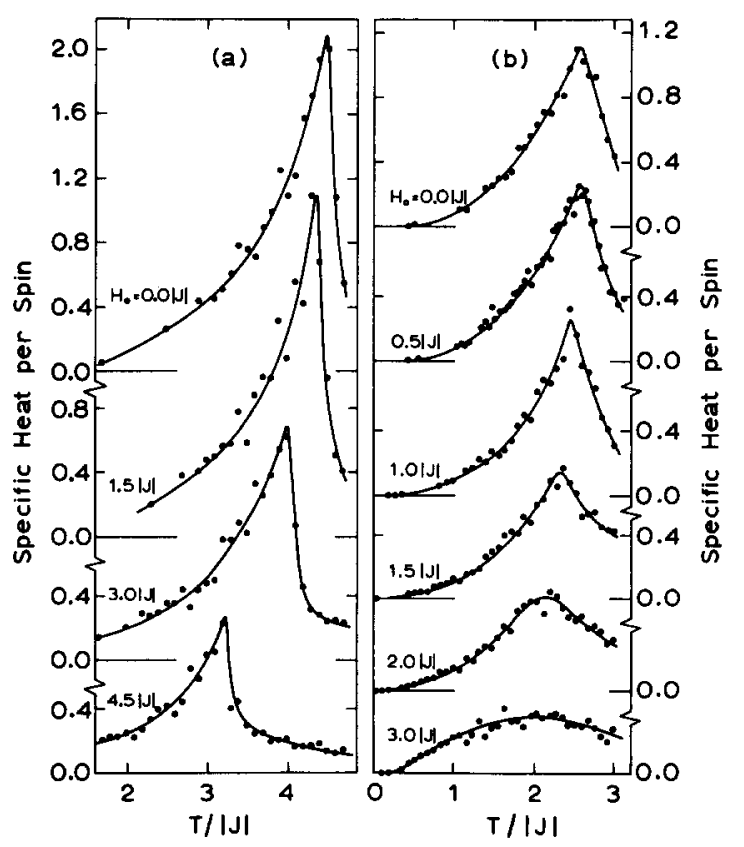

Fig. 2. Specific heat per spin as a function of temperature at various magnetic fields $H_{0}$; (a) $3 D$ pure samples; (b) $3 D$ diluted samples $(x=0.65)$. The data are obtained by averaging over four different MC runs. The solid curves are guides to the eye. 
observed in our MC simulations. Random fields are playing a minor role, as they determine only the register of the spins such that the net domain magnetization is always directed along the field. The lowering of the domain-wall energy modifies the Imry and $\mathrm{Ma}$ argument [9] and causes break-up of long-range order at high fields in arbitrarily-high dimensions.

There is a resemblance between the present domain states and those observed in experiments with pulsed high-magnetic fields on the diluted antiferromagnets $\mathrm{Fe}_{x} \mathrm{Zn}_{1-x} \mathrm{~F}_{2}$ [10] and $\mathrm{Rb}_{2} \mathrm{Co}_{x} \mathrm{Mg}_{1-x} \mathrm{~F}_{4}$ [11]. In these experiments a transition to a disordered state is observed when at low temperatures an increasing field exceeds a critical value $H_{c r}$. These transitions occur at $H_{c r} \simeq 3.5|J|$ in the $3 D \mathrm{Fe}_{x} \mathrm{Zn}_{1-x} \mathrm{~F}_{2}$ and at $H_{c r} \simeq 1.8|J|$ in the $2 D$ $\mathrm{Rb}_{2} \mathrm{Co}_{x} \mathrm{Mg}_{1-x} \mathrm{~F}_{4}$, for concentrations comparable to the ones used in our MC study. These values for $H_{c r}$ are higher than the critical fields above which domains have been observed in the simulations $\left(H_{c r} \simeq|J|\right.$ in $2 D$ and $H_{c r} \simeq 2|J|$ in $3 D$ ). Most likely, this discrepancy is caused by the order in which the field and the temperature are applied. In the MC runs thermodynamic fluctuations allow the system to find the most favourable wall configuration, in which it gradually freezes upon cooling to the lowest temperature. On the other hand, in the magnetization experiments the system is ordered antiferromagnetically at low temperature and enters the disordered state at $H_{c r}$ by a sudden flip of a large number of spins. Obviously this will occur at larger fields than in the MC simulation.

Recently a mean-field simulation of diluted Ising antiferromagnets in a field was performed by Yoshizawa and Belanger [12]. Although at low temperatures the completely antiferromagnetically ordered state has the lowest energy (at $H_{0}=0.5|J|$ and $H_{0}=|J|$ in their $15 \%$ diluted $2 D$ sample), there exists a temperature range near $T_{N}$ where a domain configuration is energetically favoured. Upon cooling through this regime this domain state is locked in. By contrast, in the present MC simulations always the state with the lower energy is reached at low temperatures. This difference must be associated with the absence of thermal fluctuations in the mean-field model.

In order to study random-field effects solely, low fields should be used. Consequently only our low-field results allow comparison to the MC findings for the RFIM [4]. Both in $2 D$ and $3 D$, our low-field simulations yield single-domain states, which are equally often registered parallel as antiparallel to the staggered anisotropy field. In this aspect our findings differ from the RFIM results [4] where similar observations are made only in $2 D$. However, the observed single-domain states are insufficient to prove the break-up of long-range order. Since no domain walls are formed within our sample, the register of the spins is determined by the gain in bulk energy only. Much larger lattices should be used to distinguish whether or not long-range order is broken up.

In conclusion, this MC work reveals that domain states are entered when cooling a diluted Ising antiferromagnet in a high magnetic field, due to the energy gain of the domain walls rather than to random-fields.

Acknowledgements - The authors are indebted to Prof. H.W. de Wijn for a critical reading of the manuscript.

\section{REFERENCES}

1. For a review see Y. Imry, J. Stat. Phys. 34, 849 (1984); G. Grinstein, J. Appl. Phys. 55, 2371 (1984).

2. I. Morgenstern, K. Binder \& R.M. Hornreich, Phys. Rev. B23, 287 (1981); K. Binder, Phys. Rev. B29, 5184 (1984).

3. D.P. Landau, H.H. Lee \& W.Kao, J. Appl. Phys. 49, 1356 (1978); E.B. Rasmussen, M.A. Novotny \& D.P. Landau, J. Appl. Phys. 53, 1925 (1982); D. Stauffer, C. Hartzstein, K. Binder \& A. Aharony, Z. Phys. B55, 325 (1984).

4. D. Andelman, H. Orland \& L.C.R. Wijewardhana, Phys. Rev. Lett. 52, 145 (1984).

5. S. Fishman \& A. Aharony, J. Phys. C12, L729 (1979).

6. P. Wong, S. Von Molnar \& P. Diman, J. Appl. Phys. 53, 7954 (1982); P. Wong, S. von Molnar \& P. Diman, Solid State Commun. 48, 573 (1983).

7. J.L. Cardy, Phys. Rev. B29, 505 (1984).

8. R.A. Cowley, R.J. Birgeneau, G. Shirane \& H. Yoshizawa, in Multicritical Phenomena (Edited by R. Pynn and A. Skjeltrop), Plenum, New York (1983).

9. Y. Imry \& S. Ma, Phys. Rev. Lett. 35, 1399 (1975).

10. A.R. King, V. Jaccarino, T. Sakakibara, M. Motokawa \& M. Date, Phys. Rev. Lett. 47, 117 (1981); J. Appl. Phys. 53, 1874 (1982); in High Field Magnetism (Edited by M. Date), p. 11. North Holland, Amsterdam (1983); J. Magn Magn. Mat. 31-34, 1119 (1983).

11. M. Motokawa, T. Harada, T. Sakakibara, M. Date, H. Ikeda \& A.R. King, J. Phys. Soc. Jpn 53, 2710 (1984).

12. H. Yoshizawa \& D.P. Belanger, Phys. Rev. B30, 5220 (1984). 